wird sie in die Enge treiben und die Völker werden sie dazu bringen, die Notwendigkeit der friedlichen Koexistenz anzuerkennen.

Am interessantesten ist, dass die UdSSR und die Länder des sozialistischen Lagers sich nicht nur der wirtschaftlichen, politischen und wissenschaftlichen Kontrolle durch die kapitalistischen Länder entzogen, sondern auch begonnen haben, mit ihnen in dieser Hinsicht in Wettbewerb zu treten. Das alles zwingt die Menschen im Westen dazu, über die Zukunft nachzudenken. Es gibt keinen anderen Weg - entweder Krieg oder friedliche Koexistenz. Wir werden niemals einen Krieg anfangen. Ich als Kommunist bin von den Vorzügen unserer Ordnung überzeugt und glaube, dass wir durch friedlichen Wettbewerb, durch unser Beispiel, durch die Entwicklung der Produktivkräfte den Sieg friedlich erringen werden.

Ich weiß, dass meine derartigen Erklärungen die Antikommunisten rasend machen. Doch mit der Zeit werden sie sich daran gewöhnen und sie [diese Erklärungen] vielleicht sogar zu loben anfangen.

Gen. Chruschtschow wünschte abschließend J. Bernal Erfolge bei seiner schwierigen, aber edlen Tätigkeit zum Nutzen des Friedens.

Aufgezeichnet von W. Michajlow Von Chruschtschow nicht durchgesehen

Übersetzt aus dem russischen Original RGANI, fond 52, opis‘ 1 , delo 552, Bl.65-74

\title{
23. Chruschtschows Rede zur Eröffnung der Beratung der Vertreter der kommunistischen und Arbeiterparteien der sozialistischen Länder in Moskau am 14. November 1957440
}

\section{Werte Genossen!}

Erlauben Sie mir, im Auftrag des Zentralkomitees der Kommunistischen Partei der Sowjetunion alle unsere lieben Freunde, die sich hier eingefunden haben, herzlich zu begrüßen.

Viele kommunistische und Arbeiterparteien haben es für notwendig gehalten, eine Beratung von Vertretern unserer Parteien durchzuführen, um einige der herangereiften Fragen der kommunistischen und Arbeiterbewegung zu erörtern. Unsere Parteien haben eine Übereinkunft darüber erzielt, eine solche Beratung in Moskau während der Feierlichkeiten zum 40.Jahrestag der Oktoberrevolution durchzuführen.

Und jetzt stellen wir alle mit großer Genugtuung fest, dass diese Beratung ihre Arbeit aufnimmt. Die heutige Beratung ist die bedeutendste Beratung der kommunistischen und Arbeiterparteien der sozialistischen Länder.

Es ist wohl kaum nötig zu betonen, welch eine große Bedeutung unsere Beratung hat. Wie wir schon vorab vereinbart haben, steht uns ein Meinungsaus- 
tausch zu einem weit gespannten Fragenkreis bevor, darunter auch zu allgemeinen Problemen der internationalen Lage sowie zu Fragen der kommunistischen und Arbeiterbewegung.

Hier sind Vertreter der kommunistischen und Arbeiterparteien zusammengekommen, die schon viele Jahre an der Spitze des Kampfes für den Aufbau des Sozialismus in ihren Ländern stehen und dabei gewaltige Erfolge erzielt haben. In unseren Ländern ist eine sozialistische Industrie geschaffen worden, es sind in allen Bereichen der Volkswirtschaft zahlreiche Kader ausgebildet und reiche Erfahrungen beim sozialistischen Aufbau gesammelt worden.

Wir müssen die Kräfte, über welche die sozialistischen Länder verfügen, richtig einsetzen, um auf dem Weg des Sozialismus und Kommunismus weiter voranzukommen. Es ist schädlich, die Kräfte des Gegners zu überschätzen, aber noch schädlicher und gefährlicher, unsere eigenen Kräfte zu unterschätzen.

Seitens unserer Parteien, seitens der sozialistischen Länder ist eine weitere Konsolidierung der Kräfte notwendig im Kampf gegen die Umtriebe der imperialistischen Reaktion und für die Stärkung der Kräfte des Sozialismus und der Demokratie, für die Bewahrung und Festigung des Friedens.

Wir, die Vertreter der kommunistischen und Arbeiterparteien der sozialistischen Länder, tragen eine besondere Verantwortung. Denn wir führen nicht nur den Kampf für die Verbreitung der Ideologie des Marxismus-Leninismus bei den Massen und für die Reinheit dieser Ideologie, sondern wir setzen auch die Ideen des Marxismus-Leninismus in die Praxis um, wir errichten die sozialistische Gesellschaft und kämpfen für den Triumph des Kommunismus. Deswegen hat unsere Arbeit Bedeutung nicht nur in theoretischer Hinsicht, wir beweisen auch in der Praxis die gewaltigen Vorzüge des Sozialismus. Das ist unsere stärkste Waffe unter dem Gesichtspunkt der Einflussnahme auf das Bewusstsein der breiten Massen der Werktätigen aller Länder.

Um im Kampf um neue Siege des Sozialismus Erfolge zu erringen, müssen die kommunistischen und Arbeiterparteien die Arbeiterklasse, die werktätige Bauernschaft und die Intelligenz um sich herum versammeln. Das Pulver muss man stets trocken halten. Und unser Pulver, das ist der Marxismus-Leninismus. Daraus folgt, dass es für uns notwendig ist, die Einheit der proletarischen Reihen in vollem Maße zu festigen, gegen Entstellungen des Marxismus-Leninismus zu kämpfen und unsere Ideologie rein zu halten.

Wir müssen gegenüber denen unversöhnlich sein, die Verwirrung in unsere Reihen tragen wollen. Die wichtigste Aufgabe besteht darin, einen entschiedenen Kampf sowohl gegen den Dogmatismus als auch insbesondere gegen den Revisionismus zu führen, der die Hauptgefahr in der gegenwärtigen Etappe darstellt.

Als Marxisten können wir dabei nicht die Augen davor verschließen, dass es in jedem unserer Länder in diesem oder jenem Umfang derartige Erscheinungen gibt, und ausgehend von den konkreten Umständen müssen wir den Kampf führen gegen die Gefahr einer Revision des Marxismus-Leninismus, gegen Versuche zur Unterminierung der Einheit der sozialistischen Länder und der Einheit der kommunistischen und Arbeiterparteien.

Genossen! Viele kommunistische und Arbeiterparteien der sozialistischen Länder haben Vorschläge unterbreitet, damit als Resultat der Beratung unserer 
Parteien ein gemeinsames Dokument verabschiedet wird, in dem die einheitliche Position unserer Parteien in den aktuellen Fragen der internationalen Entwicklung zum Ausdruck kommt. Der Entwurf eines solchen Dokuments, eine Deklaration, ist von den Zentralkomitees der Kommunistischen Parteien Chinas und der Sowjetunion unter Berücksichtigung der Vorstellungen und Wünsche der anderen Parteien gemeinsam ausgearbeitet und an alle Delegationen verschickt worden.

Die einmütige Billigung und Veröffentlichung der Deklaration wird eine überzeugende Demonstration der Einheit und Geschlossenheit der Kommunisten der sozialistischen Länder, des Kerns der gesamten kommunistischen Weltbewegung, sein. Sie wird der feindlichen antikommunistischen Propaganda einen vernichtenden Schlag zufügen und in den Reihen aller kommunistischen und Arbeiterparteien Mut und Zuversicht verbreiten.

Man darf nicht außer Betracht lassen, dass die Erfolge der Bruderparteien in den kapitalistischen Ländern, ihre Autorität und ihr Einfluss bei den breiten Massen in vielerlei Hinsicht von der Tätigkeit der Parteien der sozialistischen Länder abhängen, denn wir haben in unseren Ländern die Möglichkeit, die Überlegenheit des Sozialismus als der besseren Gesellschaftsordnung im Vergleich zum Kapitalismus praktisch vorzuführen. Mit unserer friedliebenden Außenpolitik nehmen wir Einfluss auf die gesamte internationale Lage im Interesse der Bewahrung und der Festigung des Friedens.

Die Einheit der kommunistischen Parteien der sozialistischen Länder auf der ideellen Grundlage des Marxismus-Leninismus hat gewaltige Bedeutung für die Bruderparteien der kapitalistischen Länder. Man kann es direkt sagen: Je gefestigter und geschlossener die sozialistischen Länder sind, desto enger wird die Einheit unserer kommunistischen und Arbeiterparteien, desto gefestigter und geschlossener werden die Reihen der gesamten internationalen kommunistischen Bewegung sein. Mit der einmütigen Annahme der Deklaration werden unsere Parteien, die auf den Positionen des Marxismus-Leninismus stehen, einmal mehr ihre unerschütterliche Geschlossenheit bekunden.

Genossen! Ihnen ist bekannt, dass die Repräsentanten einiger kommunistischer und Arbeiterparteien die Frage gestellt haben und weiterhin stellen, eine Beratung von Vertretern aller kommunistischen und Arbeiterparteien der sozialistischen und kapitalistischen Länder durchzuführen. Derartige Vorschläge sind sowohl beim ZK der KPdSU als auch bei den Zentralkomitees Ihrer Parteien eingegangen.

Jetzt haben sich im Zusammenhang mit den Feierlichkeiten zum 40. Jahrestag der Großen Oktoberrevolution mit wenigen Ausnahmen die Vertreter aller kommunistischen und Arbeiterparteien der Welt in Moskau versammelt. Alle Delegationen haben sich auf die Durchführung einer solchen Beratung verständigt, um gemeinsam die brennenden Fragen der Gegenwart zu erörtern, sich gegenseitig mit den Ansichten und Positionen bekannt zu machen, Erfahrungen auszutauschen und gemeinsame Aktionen in der weiteren Tätigkeit der kommunistischen und Arbeiterparteien aufeinander abzustimmen.

Von unseren Parteien ist schon eine gewisse Vorarbeit zur Vorbereitung der gemeinsamen Beratung aller Parteien in Moskau geleistet worden, und es liegt 
eine Übereinkunft mit den Bruderparteien vor über die Eröffnung dieser Beratung sofort nach dem Ende der Arbeit unserer Beratung.

Genossen, gestatten Sie mir, die Beratung der Vertreter der kommunistischen und Arbeiterparteien der sozialistischen Länder zu eröffnen und dem Wunsch nach einer erfolgreichen Tätigkeit Ausdruck zu verleihen.

Übersetzt aus dem russischen Originaltext RGANI, fond 52, opis‘ 1 , delo 443, Bl. 112-117

\section{Gespräch Chruschtschows mit Geschäftsleuten aus Kanada am 14. Mai 1958}

Am Gespräch nahmen 31 kanadische Industrielle und Persönlichkeiten der Finanzbranche teil.

Von sowjetischer Seite nahmen an dem Gespräch teil die Genossen A[nastas] I. Mikojan, I[van] G. Kabanow, Ju. E. Maksarjow.

Chruschtschow: Gestatten Sie mir, meine Herren, Sie zu begrüßen und Ihnen zuzuhören. Wir werden gerne die Sie interessierenden Fragen beantworten.

Dalgleish:441 Mit Ihnen heute zusammenzutreffen, ist uns eine große Ehre. Wir wissen, dass Sie mit wichtigen Staatsgeschäften sehr stark beschäftigt sind, und ich denke, dass wir Zeit sparen, wenn ich als Einziger der ganzen Gruppe das Gespräch führe.

Chruschtschow: Bitteschön. Wenn Ihre Kollegen mit dieser Regelung einverstanden sind, stimmen wir zu. Das riecht etwas nach Diktat, aber das ist Ihre Sache (allgemeine Heiterkeit).

Dalgleish: Wir, 30 Kanadier, die in die Sowjetunion gekommen sind, haben viel gesehen und mit vielen Leuten gesprochen. Wir haben viele Ihrer Errungenschaften gesehen, und ich würde sagen, einer der Umstände, der uns tief beeindruckt hat, war die Tatsache, dass die Probleme, die vor den Sowjetmenschen stehen, sehr den Problemen ähneln, die vor den Völkern anderer Länder stehen. Und ich muss sagen, wir stimmen den Aussagen Ihrer Leute darüber zu, dass wir, die Kanadier, und Sie einander ebenso gute Nachbarn sein sollten, wie wir es mit den Vereinigten Staaten von Amerika sind. Wie denken Sie, Sir, ließe sich das am besten erreichen?

Chruschtschow: Ich möchte die Frage präzisieren: Sie haben gute, freundliche Beziehungen mit den USA? Sie hätten gern ebensolche Beziehungen mit uns?

Dalgleish: Von Sowjetmenschen haben wir viele Male den Wunsch vernommen, dass unsere Beziehungen mit Ihnen ebenso gut sein sollten, wie es unsere Beziehungen mit den USA sind.

${ }^{441}$ Oakley Dalgleish, Herausgeber der Zeitung „The Globe and Mail“ in Toronto. 